\title{
Desafios na implantação de boas práticas na Central de Material e Esterilização e a segurança do paciente
}

\author{
Challenges in implementing good practices in the Material and Sterilization Center and \\ patient safety
}

\author{
Desafíos en la implementación de buenas prácticas en el Centro de Material y \\ Esterilización y seguridad del paciente
}

Silvana Silva de Souza ${ }^{1}$, Samara Bianca Silva da Silva², Maria José do Nascimento Silva², Lucrecia Aline Cabral Formigosa ${ }^{3 *}$.

\section{RESUMO}

Objetivo: Analisar os desafios enfrentados pelo enfermeiro na Central de Material e Esterilização (CME) no processamento de artigos, com foco na cirurgia segura. Métodos: Estudo transversal, com abordagem qualitativa, realizada em hospital público de ensino em Belém/PA/Brasil, tendo como participantes enfermeiros da CME. A coleta de dados foi realizada através de questionário, com perguntas abertas, após a assinatura do Termo de Consentimento Livre e Esclarecido. Resultados: A partir da análise dos discursos, emergiram três categorias, a saber: Os Desafios da Implementação das Boas práticas de Processamento de Produtos para a Saúde; Relação da CME para a Cirurgia Segura; e a Capacitação Profissional como fator para a Segurança do Paciente. Conclusão: $O$ estudo possibilitou identificar que os principais desafios enfrentados pelos enfermeiros da CME são estrutura física inadequada, falta de insumos, escassez de recursos humanos, falta de protocolos e capacitação da equipe. Compreender tais dificuldades é o primeiro passo para a mudança de cultura em relação às Boas Práticas de Processamento de Produtos para a Saúde.

Palavras-chave: Enfermagem, Esterilização, Segurança do paciente.

\begin{abstract}
Objective: To analyze the challenges faced by nurses at the Material and Sterilization Center (MSC) in the processing of articles, with a focus on safe surgery. Methods: This was cross-sectional study, with a qualitative approach, carried out in a public teaching hospital in Belém / PA / Brazil, with participants from MSC nurses. Data collection was performed through a questionnaire, with open questions, after signing the informed consent form. The study was approved by the Research Ethics Committee. Results: From the analysis of the speeches, three categories emerged, namely: The Challenges of Implementing Good Practices for Processing Products for Health; List of MSC for Safe Surgery; and Professional Training as a factor for Patient Safety. Conclusion: It is made possible to identify that the main challenges faced by the nurses of the MSC are inadequate physical structure, lack of inputs, shortage of human resources, lack of protocols and training of the team. Understanding such difficulties is the first step towards changing culture in relation to Good Practices for Processing Health Products.
\end{abstract}

Keywords: Nursing, Sterilization, Patient safety.

${ }^{1}$ Empresa Brasileira de Serviços Hospitalares (EBSERH), Belém - PA.

2 Faculdade Pan Amazônica (FAPAN), Belém - PA.

3 Universidade Federal do Pará (UFPA), Belém - PA. *E-mail: lucrecia_cabral@hotmail.com 


\section{RESUMEN}

Objetivo: Analizar los desafíos que enfrentan las enfermeras en el Centro de Material y Esterilización (CME) en el procesamiento de artículos, centrándose en una cirugía segura. Métodos: Se trata de un estudio transversal, con abordaje cualitativo, realizado en un hospital público de enseñanza en Belém / PA / Brasil, con enfermeras de la CME como participantes. La recolección de datos se realizó a través de un cuestionario, con preguntas abiertas, después de firmar el formulario de consentimiento libre e informado. Resultados: A partir de análisis de los discursos, emergiram tres categorias, a saber: Los desafíos de implementar buenas prácticas de procesamiento de productos para la salud; Relacion de CME con una Cirurgía Segura; y la formación profesional como factor de seguridad del paciente. Conclusión: Se concluye que los principales desafíos que enfrentan las enfermeras del CME son la estructura física inadecuada, la falta de insumos, la escasez de recursos humanos, la falta de protocolos y la capacitación del equipo. Entender tales dificultades es el primer paso para cambiar la cultura en relación con las Buenas Prácticas para Procesar Productos de Salud.

Palabras clave: Enfermería, Esterilización, Seguridad del Paciente.

\section{INTRODUÇÃO}

O desenvolvimento das técnicas cirúrgicas tornou-se mais sofisticadas e complexas, devido ao avanço tecnológico, consequentemente os instrumentos cirúrgicos tornaram-se também de maior complexidade, havendo então a necessidade de aprimoramento no tratamento desses instrumentos e a qualificação do profissional envolvido no desenvolvimento dessas tarefas para os cuidados especiais com esses materiais, para a segurança do paciente cirúrgico nos períodos trans ou pós-operatório (GOMES DR, et al., 2013).

A Central de Material e Esterilização (CME) é um desses locais e se caracteriza como um setor de serviços de saúde, inserido ou não em uma organização de saúde, devido à possibilidade de existir como uma empresa independente, prestadora de serviços de esterilização.

É um ambiente de alta concentração de equipamentos e materiais, que apresenta um processo de trabalho específico, no intuito de contribuir à qualidade dos serviços prestados por suas unidades consumidoras, por destinar-se a oferecer materiais esterilizados em condições seguras de uso (TAUBE SAM, 2007).

Quanto ao profissional envolvido no processamento, o enfermeiro é o responsável técnico pela CME, desempenhando atividades de cunho assistencial, a exemplo do preparo de materiais e priorização de atendimento de determinada área-fim, quanto de caráter gerencial, como a administração do quadro de funcionários e a provisão de insumos e equipamentos (OURIQUES CM e MACHADO ME, 2013).

Em se tratando da segurança do paciente cirúrgico, Ferraz EM (2009) afirma que a cirurgia teve um grande progresso nos últimos 50 anos, porém a infecção hospitalar continua sendo um grave problema de saúde pública, e dentre eles a infecção do sitio cirúrgico, que traz à tona a qualidade da prestação de cuidados e ocasiona o aumento do tempo de internação, a mortalidade, a morbidade e gastos hospitalares. Neste contexto, em 2009, a Organização Mundial de Saúde (OMS) iniciou uma campanha mundial, intitulada Cirurgia Segura Salva Vidas, visando reduzir o índice de erros e infecções.

De acordo com o Ministério da Saúde (2013), o protocolo de cirurgia segura vem como uma estratégia para a redução dos riscos de incidentes cirúrgicos e deverá ser aplicado em todos os estabelecimentos em que ocorrerem procedimentos que impliquem em incisão no corpo humano, determinando medidas a serem adotadas para a redução de ocorrências e eventos adversos e mortalidade relacionados à procedimentos cirúrgicos, por meio da lista de verificação de cirurgia segura.

Portanto, a lista de verificação de segurança cirúrgica da OMS foi desenvolvida para ajudar as equipes cirúrgicas a reduzira ocorrência de dados ao paciente e se divide em três fases, cada uma correspondendo a um período de tempo de um procedimento cirúrgico (ANVISA, 2012). 
Para equipe de enfermagem da CME, esse momento é um dos mais importantes, pois é quando há confirmação da esterilização, mostrando se o processamento dos artigos foi realizado com qualidade. $O$ enfermeiro no Centro Cirúrgico ou circulante define o equipamento para o procedimento cirúrgico, confirmando verbalmente que esterilização foi realizada e que o indicador do processo de esterilização comprovou a esterilização bem-sucedida. Qualquer diferença entre o resultado esperado e o resultado real do indicador do processo de esterilização deve ser comunicada a todos os membros da equipe e aborda antes da incisão (OMS, 2014).

Pancieri AP, et al. (2013) apontam que são muitos os fatores que podem levar uma equipe de cirurgia ao erro, colocando em risco a segurança do paciente, dentre eles encontram-se os materiais inadequados, por esterilização inadequada ou por mau funcionamento.

Percebe-se que muitos são os desafios do enfermeiro gerente da CME, pois tem a responsabilidade de gerenciar profissionais capacitados e habilitados com os serviços realizados nesta unidade. A supervisão irá refletir e influenciar diretamente no trabalho da equipe para a qualidade da prática segura do paciente cirúrgico. Os gestores responsáveis por estas equipes deverão buscar aquisição de novos conhecimentos através da capacitação e educação continuada (MORYIA GAA e TAKEITI MH, 2016).

A partir dessa perspectiva, emergiu a seguinte questão norteadora: Que desafios o enfermeiro da CME enfrenta para garantir uma cirurgia segura? Tendo isso em vista, o estudo objetivou analisar os desafios enfrentados pelo enfermeiro na Central de Material e Esterilização no processamento de artigos, visando à cirurgia segura.

\section{MÉTODOS}

Trata-se de pesquisa transversal, com abordagem qualitativa, exploratória, descritiva e de campo, desenvolvida em Belém do Pará, no período de julho de dezembro de 2018. Foram atendidos os preceitos éticos e a Resolução nº466/2012 do Conselho Nacional de Saúde do Ministério da Saúde, que dispõe sobre Diretrizes e Normas Regulamentadoras para pesquisas envolvendo seres humanos, sendo iniciada após a aprovação do projeto pelo Comitê de Ética em Pesquisa da Universidade Paulista (UNIP) sob o protocolo no CAAE 96056918.3.0000.5512.

O cenário da pesquisa foi a Central de Material e Esterilização (CME) de um hospital público, destinando a totalidade da capacidade instalada ao SUS. Escolhido por ser de grande porte, atendendo a demanda de todo o Estado do Pará; e habilitado como Centro de Assistência de Alta Complexidade em Oncologia e hospital de ensino pelo Ministério da Saúde.

Utilizaram-se como critérios de inclusão: possuir graduação em enfermagem, com especialização e/ou atuação na CME, estar no exercício de suas funções e aceitar participar da pesquisa. E como critérios de exclusão: estar afastado da unidade por motivo de férias, licença à saúde e outros afastamentos legais, e não se disponibilizar a participar da pesquisa.

Para a coleta de dados, primeiramente, obteve-se junto à instituição a relação dos profissionais que atendiam aos critérios de inclusão, a fim de que houvesse uma aproximação com os mesmos. Em seguida, realizou-se uma abordagem prévia individual, para apresentação da proposta da pesquisa e obtenção do aceite de participação, procedendo-se a leitura e assinatura do Termo de Consentimento Livre Esclarecido (TCLE).

Como instrumento de obtenção dos dados foi empregado questionário, elaborado em duas partes: a primeira visando caracterizar a população do estudo, incluindo-se as variáveis: faixa etária, gênero, nível de formação, tempo de formado, tempo de atuação na CME e turno de trabalho abertas; e a segunda com questões abertas que levariam os profissionais a refletirem sobre os desafios enfermeiros da CME.

Os próprios sujeitos da pesquisa efetuaram o preenchimento dos questionários, com intuito de não interferir, nem tampouco intimidá-los nas respostas. Após, houve o agendamento de hora, lugar e dia para recebimento dos mesmos devidamente preenchidos, conforme a disponibilidade dos próprios profissionais, para que não houvesse interrupção no desempenho de suas funções laborais. 
A amostra do estudo foi composta por dez enfermeiros dos três turnos de trabalho: matutino, vespertino e noturno. Para análise dos dados, foi utilizada a técnica de análise de conteúdo, caracterizada por buscar o entendimento dos diversos significados com base nas realidades visível e invisível ("entrelinhas" do texto), no qual as respostas serão descritas, mediante as fases de pré-análise, exploração, tratamento e interpretação dos resultados, correlacionando-os com a produção científica sobre os desafios dos enfermeiros da CME (MINAYO MCS, 2012).

Da análise, emergiram três categorias: Desafios para a Implantação das Boas Práticas de processamento de produtos para a saúde; Contribuição da CME para a cirurgia segura; e, Capacitação profissional como fator para a segurança do paciente. Os dados quantitativos foram transcritos ao Microsoft Office Excel® 2016, e organizados em tabela para melhor descrição dos mesmos.

\section{RESULTADOS}

O estudo contou com a participação de 10 profissionais enfermeiros, com predominância do gênero feminino ( $100 \%$ dos participantes), com $80 \%$ deles na faixa etária de 41 a 50 anos. Em relação ao campo de atuação, $80 \%$ eram assistenciais e $20 \%$, gestores. Todos possuíam pós-graduação lato sensu. $O$ tempo de formação foi de 5-10 anos para 60\% dos entrevistados. No que se refere ao tempo de atuação na CME, variou de 1 até 8 anos, sendo que $40 \%$ atuavam no setor entre 2 a 4 anos.

Quanto ao horário de trabalho, $40 \%$ exerciam as atividades laborais no período matutino, assim como no noturno (40\%), entretanto, nesse período o profissional enfermeiro não era exclusivo da CME, atuando também no centro cirúrgico. Na Tabela 1 são apresentadas as características sociodemográficas dos participantes da pesquisa.

Tabela 1 - Caracterização sociodemográficas dos profissionais participantes do estudo.

\begin{tabular}{lcc}
\hline Variáveis & N & $\%$ \\
\hline Gênero & 10 & 100 \\
\hline Feminino & 0 & 0 \\
Masculino & 2 & 20 \\
\hline Faixa Etária & 8 & 80 \\
\hline 31 a 40 anos & & 80 \\
41 a 50 anos & 8 & 20 \\
\hline Campo de atuação & 2 & \\
\hline Assistência & & 0 \\
Gerência & 0 & 100 \\
\hline Nível de formação & 10 & 60 \\
\hline Somente Graduação & & 20 \\
Pós-graduação & 6 & 20 \\
\hline Tempo de formação & 2 & 40 \\
\hline $5-10$ anos & 2 & 40 \\
$11-20$ anos & & 20 \\
Acima de 21 anos & 4 & \\
\hline Tempo de atuação na CME & 4 & 20 \\
\hline $0-1$ anos & 2 & 40 \\
$2-4$ anos & & \\
Acima de 5 anos & 4 & 2 \\
\hline Turno de trabalho & 4 & \\
\hline Matutino & & \\
Vespertino & & \\
Noturno & & \\
\hline Fonte: Souza SS, et al., & & \\
\hline
\end{tabular}

Fonte: Souza SS, et al., 2020 
Quando perguntados sobre os desafios ou dificuldades enfrentadas no cotidiano para a implantação de boas práticas de processamento de artigos para saúde, os respondentes colocaram como desafios: a estrutura física inadequada, falta de insumos para os procedimentos cirúrgicos e procedimentos em clínicas e equipamentos necessários para o processo de limpeza dos produtos. Tais aspectos podem ser observados na fala a seguir descrita:

"[...] quanto à equipe, percebe-se a falta de atenção na realização do processo, visto que os servidores precisam ter consciência da importância dos serviços realizados, sendo contínuo e rotineiro, devendo ser exercido com responsabilidade, segundo as normas recomendadas pela RDC 15 [...]" (E1)

Outro desafio mencionado pelos profissionais foi:

"[...] Falta de insumos adequados para o ano todo, estrutura física péssima." (E2).

Os participantes também mencionam que:

"[...] Ainda esbarramos na falta de pessoas e equipamentos, que por seu uso constante acabam por apresentar problemas técnicos com frequência" (E7).

Em relação à questão se considera a CME importante para a segurança do paciente e como contribui para tanto, os participantes da pesquisa responderam que a CME é fundamental, uma vez que por meio do processo de trabalho realizado durante a esterilização, assegurado através de protocolos, garante-se 0 mínimo para o procedimento cirúrgico seguro, conforme observado na seguinte fala:

"Sim, a CME contribui processando materiais, artigos de acordo com a RDC 15, realizando boas práticas na segurança do paciente" (E5).

De modo semelhante, percebe-se o discurso de E3 quando afirma:

"Com certeza (importância da CME para a segurança do paciente), pois a eficácia do processo de esterilização de todos os materiais médico hospitalares que são utilizados no paciente evita danos ao mesmo [...]".

Para o respondente E8, a relação entre CME e cirurgia segura é:

"Sem dúvida é de suma importância, visto que, em seu processo de esterilização de materiais que serão utilizados em procedimentos invasivos são comprovados através dos testes biológicos realizados e dos integradores que comprovam seu processo".

Outro apontamento dos participantes do estudo foi a importância da capacitação direcionada à assistência baseada em políticas de segurança do paciente para a CME, pois somente desse modo serão conscientizados quanto à necessidade de se desenvolver os processos adequadamente para minimizar eventos adversos.

Nesse sentido, foi expresso por um dos sujeitos da pesquisa, que:

"O profissional deve ser capacitado para desenvolver suas tarefas, agindo com responsabilidade e compromisso [...]" (E6).

$\mathrm{O}$ que foi corroborado pelo participante $\mathrm{E} 10$ quando afirma que:

"Toda equipe deve ser capacitada na segurança do paciente, desta forma a equipe realizará suas atividades e rotinas com segurança".

A despeito de reafirmar a premente necessidade de capacitação, um dos respondentes frisou a presença em cursos de aperfeiçoamento constitui iniciativas isoladas:

"Sim, porém aqui ainda é muito tímida essa capacitação, embora a maioria já tenha consciência". (E4) 


\section{DISCUSSÃO}

A caracterização da população do estudo aponta que a enfermagem ainda majoritariamente é feminina, o que pode ser explicado pela construção sócio-histórica da profissão, onde o cuidado no espaço formal das relações de trabalho na saúde necessita de atributos inerentes às mulheres (LOPES MJM e LEAL SMC, 2005).

É importante mencionar que o trabalho desenvolvido na CME exige muito esforço físico, especialmente para o carregamento de instrumentais cirúrgicos, o que pode causar lesões físicas pelo excesso de utilização da força muscular em mulheres (REGO GMV, et al., 2020).

A faixa etária dos participantes da pesquisa demonstra uma população adulta madura (41 a 51 anos), concordante com a estimativa do Instituto Brasileiro de Geografia e Estatística (2016) que prevê maior número de pessoas economicamente ativas na faixa etária de 25 a 49 anos.

Para $60 \%$ dos entrevistados, o tempo de formação foi entre 5-10 anos e todos possuíam pós-graduação. Contudo, percebe-se pouco tempo de atuação no setor (2 a 4 anos). Nesse sentido, ainda que exista a carência de vivência experienciada na prática, hoje o corpo de enfermagem está muito mais qualificado para atuar nesta área. Ressalte-se que, o profissional, individualmente, não irá alcançar os progressos necessários se não houver engajamento institucional neste sentido (BUGS TV, et al., 2017).

Quanto ao turno de trabalho, $60 \%$ atuavam durante o diurno (manhã e tarde) e $40 \%$ trabalhavam no noturno, sendo que neste período o enfermeiro atua conjuntamente na CME e no Centro Cirúrgico. A RDC 15, de 2012, em seu artigo 28, parágrafo único, estabelece que o responsável pela CME, classe II, deve atuar exclusivamente nesta unidade durante sua jornada de trabalho (ANVISA, 2012).

No que tange aos desafios para a implementação de boas práticas em CME, é fundamental que possa tornar o trabalho visível e reconhecido pela instituição e pelos outros profissionais. Para tanto, é necessário demonstrar que o trabalho em CME não se limita a uma simples limpeza de materiais, mas requer conhecimentos específicos para serem executados.

Concomitante a isso, o profissional precisa ter compromisso e responsabilidade com a organização, reposição, diminuição de custos e gerenciamento dos materiais médico-cirúrgicos, buscando reduzir parte dos custos perioperatório em que a esterilização excessiva de instrumentais cirúrgicos, muitas vezes, é desnecessária (SANCHEZ MA, 2018).

Baseando-se nesse prisma, depreende-se que são inúmeros os desafios. Os mais destacados nas falas dos participantes foram: falta de estrutura física, equipamentos sem manutenção, recursos humanos insuficientes e a inexistência de protocolos.

Em muitos casos, a inadequação arquitetônica da CME está relacionada à pouca valorização e visibilidade do setor junto a gestores e profissionais, sendo relegado à localização que não atende as demandas e/ou sendo agregado equivocadamente ao CC. Ademais, o desempenho dos profissionais nessa unidade revela a percepção da importância e destaque dessa unidade com a responsabilidade no processamento de materiais (ATHANÁZIO AR e CORDEIRO BC, 2015).

Reforçam Silva LSL, et al. (2020) que a assistência segura aos usuários se interliga à estrutura física da CME, pois localização e dimensionamento correto poderão proporcionar a eficácia e segurança nas atividades desenvolvidas, tais como: receber, desinfetar e separar os produtos para saúde; lavar os produtos; receber as roupas vindas da lavanderia; preparar os produtos e roupas (pacotes); esterilizar os artigos para todos os setores do hospital, por meio de métodos físicos, químicos ou físico-químicos; realizar o controle microbiológico e prazo da validade de esterilização dos produtos esterilizados; armazenar e distribuir os artigos de saúde e as roupas esterilizadas.

As dificuldades relacionadas à inadequação dos equipamentos ou escassez de recursos materiais, como as autoclaves, lavadoras, termodesinfetadora, podem gerar sentimentos de insatisfação, fadiga e resiliência nos profissionais, prejudicando o comprometimento com o trabalho realizado (PARENTE AN, et al., 2018). 
Ressaltou-se a importância dos Protocolos Operacionais Padrão (POP), para a sistematização do processo realizado na CME. Sales CB, et al. (2018) reforçam as falas ao afirmar ser o POP uma ferramenta gerencial para enfermeiro, a fim de padronizar as intervenções de enfermagem, devendo ser construída juntamente com a equipe.

Além de apoiar a tomada de decisão do enfermeiro, possibilita também corrigir as não conformidades, diminuindo as distorções adquiridas na prática, tendo finalidade educativa. Sua adoção proporciona satisfação para a equipe e pacientes, pela maior segurança na realização dos procedimentos e consequentemente maior segurança ao paciente.

De acordo com Souza RQS, et al. (2020), o trabalho do enfermeiro na manutenção, validação e controle de rotina dos métodos esterilizantes, e na qualificação e identificação das necessidades de sua equipe quanto às suas dúvidas sobre o processo de trabalho na CME garante a eficácia dos processos, além de contribuir para a prevenção de infecções hospitalares. Portanto, aos enfermeiros coordenadores da CME, compete participar do dimensionamento e da definição da qualificação necessária aos profissionais para a atuação no local.

A relação CME e Cirurgia Segura ficou evidente no discurso dos participantes, pois realiza todo o processo de esterilização e garante a eficácia de cada artigo processado através do monitoramento por meio de indicadores, garantindo a segurança do paciente.

Existem vários aspectos capazes de contribuir para a ocorrência de erros no período peri-operatório, o que deixa a segurança e a qualidade no atendimento do paciente vulneráveis. Exemplos claros desses fatores são: identificação incorreta do material, impossibilitando o rastreabilidade do ciclo; instrumentais danificados após o processo de esterilização, tornando-os impróprios ao uso (PANCIERI AP, et al., 2013).

Enfatiza-se que atitude de cada colaborador que atua na CME e o trabalho de supervisão do enfermeiro reflete e influência diretamente na execução de prática segura ao paciente cirúrgico, mesmo que o cuidado seja indireto, garantindo a reprodutibilidade do processo em sua totalidade. Essas atitudes permeiam a possibilidade do rastreamento de todas as fases do processamento de materiais no que tange ao controle de infecção hospitalar no caso de um necessário recall de produtos para a saúde (MORIYA GAA e TAKEITI MH, 2016).

Outro ponto destacado nos discursos foi a importância da capacitação para atuação em CME, pois propicia desenvolver um processo seguro e com consciência científica dos profissionais. É fundamental que tanto enfermeiro quanto técnicos de enfermagem sejam capacitados para o desempenho das funções para que todas as etapas do processo sejam executadas de forma segura, lembrando que os recursos humanos são indispensáveis para a qualidade.

Nessa perspectiva, a educação permanente configura como uma ferramenta de qualificação dos profissionais de enfermagem da CME, permitindo a mudança no ambiente de trabalho, pois favorece 0 desenvolvimento cognitivo; contribui para a qualificação e satisfação laborais; aumenta o rendimento nas atividades realizadas; e, os resultados são visíveis para a assistência prestada (ATHANÁZIO AR e CORDEIRO BC, 2015).

Acrescenta-se que os sujeitos do estudo reconhecem a necessidade de capacitação em segurança do paciente, no entanto, ainda são excluídos do processo de construção dos protocolos de segurança do paciente, e especificadamente, o protocolo de cirurgia segura.

Nascimento MVF, et al. (2018) corrobora que as maiores cooperações da assistência de enfermagem são dessas equipes, visto que fazem a CME funcionar da melhor forma possível e o trabalho na unidade de saúde fluir cada vez mais, garantindo a reprodutibilidade do processo em sua totalidade. Desta feita, é notório o papel primordial que a educação continuada apresenta, porquanto evita incorreções no processo de trabalho ao permitir a reflexão das práxis diárias. 


\section{CONCLUSÃO}

O estudo possibilitou identificar que os principais desafios enfrentados pelos enfermeiros da CME são estrutura física inadequada, falta de insumos, escassez de recursos humanos, falta de protocolos e capacitação da equipe, deixando claro que mudar somente a estrutura física não resolve. Ademais, o estudo revelou a importância do setor e a necessidade de qualificação para atender demandas tão específicas, além de proporcionar maior visibilidade da CME. Ressalte-se que, apesar de o avanço da tecnologia ter facilitado o trabalho dos profissionais com a automatização das tarefas, não dispensa a atividade humana, que continua sendo fator primordial em relação à segurança dos processos de esterilização. Destarte, os resultados podem contribuir para a compreensão das dificuldades, primeiro passo para a mudança de cultura em relação às boas práticas de processamento de produtos para a saúde.

\section{REFERÊNCIAS}

1. AGÊNCIA NACIONAL DE VIGILÂNCIA SANITÁRIA (ANVISA). Resolução da Diretoria Colegiada RDC n. 15, de 15 de março de 2012. Dispõe sobre requisitos de boas práticas para o processamento de produtos para saúde e dá outras providências. Brasília, DF: Agência Nacional de Vigilância Sanitária; 2012; 18 p.

2. ATHANÁZIO AR, CORDEIRO BC. Educação permanente a trabalhadores da central de material e esterilização. Rev Enferm UFPE, 2015; 9(6): 8758-8761.

3. BRASIL. Ministério da Saúde. Anexo 03: Protocolo para Cirurgia Segura. Brasília: Ministério da Saúde; Agência Nacional de Vigilância Sanitária; Fiocruz; 2013.

4. FERRAZ EMA. Cirurgia segura: uma exigência do século XXI. Revista Colégio Brasileiro de Cirurgiões, 2009; 36(4): 281-282.

5. GOMES DR, et al. O papel da enfermeira no processo de atualização profissional dos funcionários da central de material e esterilização. Textura, 2013; 6(12): 95-102.

6. INSTITUTO BRASILEIRO DE GEOGRAFIA E ESTATÍSTICA (IBGE). Indicadores IBGE: pesquisa mensal de emprego janeiro 2016. Rio de Janeiro: IBGE; 2016; 30 p.

7. LOPES MJM, LEAL SMC. A feminização persistente na qualificação profissional da enfermagem brasileira. Cadernos pagu, 2005;(24): 105-125.

8. BUGS TV, et al. Perfil da equipe de Enfermagem e percepções do trabalho realizado em uma central de materiais. Rev Min Enferm, 2017;21(1): e-996.

9. MINAYO MCS. Análise qualitativa: teoria, passos e fidedignidade. Ciência \& Saúde Coletiva, 2012; 17(3):621-626.

10. MORIYA GAA, TAKEITI MH. O trabalho da enfermagem em Centro de Material e Esterilização e sua implicação para a segurança do paciente. Rev. SOBECC, 2016; 21(1): 1-2.

11. NASCIMENTO MVF, et al. Aspectos Assistenciais da Central De Material e Esterilização com Foco no Protocolo de Cirurgia Segura. Brazilian Journal of Surgery and Clinical Research, 2018; 24(1): 122-126.

12. ORGANIZAÇÃO MUNDIAL DA SAÚDE (OMS). Manual de Implementação Lista de Verificação de Segurança Cirúrgica da OMS 2009 - Cirurgia Segura Salva Vidas. Brasília: Organização Pan-Americana da Saúde; Agência Nacional de Vigilância Sanitária; 2014; 20 p.

13. OURIQUES CM, MACHADO ME. Enfermagem no Processo de Esterilização de Materiais. Texto \& Contexto Enfermagem, 2013; 22(3): 695-703.

14. PANCIERI AP, et al. Checklist de cirurgia segura: análise da segurança e comunicação das equipes de um hospital escola. Revista gaúcha de enfermagem, 2013; 34(1): 71-78.

15. PARENTE AN, et al. Evidências profissionais de enfermeiros em Central de Material e Esterilização: contribuições de uma revisão integrativa. Enfermagem Brasil, 2018; 17(4): 377-385.

16. REGO GMV, et al. Qualidade de vida no trabalho numa central de materiais e esterilização. Rev. Bras. Enferm, 2020; 73(2): 1-7.

17. SALES CB, et al. Protocolos Operacionais Padrão na prática profissional de Enfermagem: utilização, fragilidades e potencialidades. Rev. Bras. Enferm, 2018; 71(4): 126-134.

18. SANCHEZ MA, et al. Estratégias que contribuem para a visibilidade do trabalho do enfermeiro na Central de material e esterilização. Texto Contexto Enferm, 2018;27(1): 2-9

19. SILVA LSL, et al. (Des)conformidade do processo de trabalho no centro de material e esterilização. Rev. SOBECC, 2020; 25(1): 3-10.

20. SOUZA RQS, et al. Validação da limpeza de produtos para saúde no cotidiano do Centro de Material e Esterilização. Rev. SOBECC, 2020; 25(1): 58-64.

21. TAUBE SAM. O processo de trabalho da enfermeira na central de material e esterilização: uma perspectiva tecnológica aos instrumentos. Acta paul. enferm, 2007; 20(4): 470-475. 